\title{
Ein Blick auf die Forschungsgeschichte und Methodik der Wortfelduntersuchungen
}

\author{
József TótH \\ Lehrstuhl für deutsche Sprache und Literatur, Hochschule "Dániel Berzsenyi", \\ Szombathely, Ungarn \\ Lehrstuhl für germanistische Linguistik, Germanistisches Institut, Universität Vesz- \\ prém, Ungarn
}

The present study makes an attempt to outline the continuity of the research into word fields, pointing out its six stages and highlighting the significance of this topical issue. Since an onomasiologic collection of verbal components of word fields allows no insight into the meaning structures of the verbs in either languages, and in an effort to explore new ways of their adequate analysis, the paper is therefore trying to provide an overview of and demonstrate how modern theories of word semantics such as componential analysis and the prototype theory contribute to the further development of word field theory. I illustrate the findings of my research by using the verbal word field components 'Geschehen'/'happening'. I elaborate on the definitions of word fields, notion circle and happening, and I also touch upon the working methods employed in the course of the interlingual analysis of German and Hungarian verbs, which is then followed by an interligual semantic analysis. The use of word fields in language learning and in lexicographical research is a pivotal issue if one looks into the topic. In the case of the latter one, I point out the necessity to compile a GermanHungarian, Hungarian-German dictionary of verbal word fields.

\section{VORBEMERKUNGEN}

Anregungen zu meiner Forschungsarbeit auf dem Gebiet der Wortfeldtheorie kamen zunächst aus der langjährigen Unterrichtspraxis und aus den Unzulänglichkeiten der Beschreibung und Darstellung der lexikalischen Bedeutung in ein- und zweisprachigen Wörterbüchern. $\mathrm{Zu}$ dieser Forschungsarbeit haben aber auch die nicht immer zuverlässigen Deskriptionsmodelle in einigen linguistischen Arbeiten zu den deutschen 'geschehen'-Verben in bedeutendem Maße beigetragen.

Gegenstand der synchronischen Untersuchungen sind lexikalische Einheiten zweier genetisch nicht verwandter und auch typologisch unterschiedlicher Sprachen, genauer gesagt, die sogenannten 'geschehen'-Verben. Im Mittelpunkt des Interesses stehen sowohl die Entstehungs- und Entwicklungsgeschichte des vieldiskutierten Wortfeldbegriffes in der Sprachwissenschaft als auch die Beschreibung der Bedeutungsstruktur des verbalen Wortfeldes 'Geschehen/történés'.

Wortfelder stellen für die lexikalische Semantik besonders geeignete Untersuchungs- 
objekte dar. Wortfelder ermöglichen nämlich - nach einer semantischen und teilweise auch syntaktischen Analyse -, die semantische Breite des Wortschatzes zu verdeutlichen und seine Feinheiten bewusst zu machen. Die effektive Beschäftigung mit dem Wortschatz erfordert in erster Linie eine kohärente intralinguale linguistische Beschreibung der Glieder von Wortfeldern, aus der selbstverständlich auch der Lexikograph Schlüsse ziehen kann. Zur Bestimmung und Vermittlung der genauen Bedeutungen und des Gebrauchs einzelner Lexeme können auch synchronische interlinguale Untersuchungen beitragen. Kontrastiv angelegte Wortfeldanalysen, die bedeutungserschließende Einsichten vermitteln, haben den Vorteil, dass sie nicht nur Einblicke in die Strukturierung der Fremdsprache, sondern auch in die der Muttersprache gewähren. Ich habe daher im Laufe meiner Forschungsarbeit folgende Zielsetzung: die Kontinuität der Wortfeldforschung darzulegen, ihre Aktualität nachzuweisen, die Relevanz einiger zeitgenössischer wortsemantischer Konzeptionen für die Weiterentwicklung der Wortfeldtheorie und die Anwendbarkeit ihrer Ergebnisse an einigen verbalen Feldgliedern des Geschehens zu dokumentieren. Nach der Zusammenstellung des deutschen und ungarischen Wortmaterials ('Geschehen'/'történés') wird in einem Teilfeld eine intralinguale und dann eine kontrastive interlinguale semantische Analyse durchgeführt. Als Grundlage für die intralinguale Bedeutungsbeschreibung und die deutsch-ungarische Konfrontation dient eine von mir bevorzugte Methode. Die Ergebnisse der Forschungsarbeit dienen dazu, die sprachlichen Nuancierungen und Differenzierungen auf der lexikalischsemantischen und syntaktischen Ebene in der deutschen und ungarischen Sprache zu erfassen.

\section{Methodische Vorgehensweise und Aufbau der Forschungsarbeit}

Ausgehend von den Denkanstößen für die Lehre vom sprachlichen Feld (Hoberg 1970) untersuche ich im Laufe meiner Forschungsarbeit die unterschiedlichsten und umstrittenen Wortfeldkonzeptionen und überprüfe sie hinsichtlich ihrer Tauglichkeit bei der Wiedergabe der lexikalischen Struktur der Sprache. Dabei werden vor allem ihre Stärken aber auch ihre Einseitigkeiten hervorgehoben. Im Brennpunkt stehen einerseits ihre Entwicklungsphasen in den früheren Zeiten, das Nachwirken der Wortfeldtheorie(n) in der strukturellen Semantik und ihre weiteren Ausprägungsvarianten sowie die Relevanz zahlreicher zeitgenössischer wortsemantischer Konzepte für die Weiterentwicklung der Wortfeldthoerie (der Wortfeldbegriff von Lutzeier 1981, die Komponential- und die Prototypensemantik). Da die interlingual-kontrastive Analyse besonders geeignet ist, unbewusste sprachimmanente Bedeutungsstrukturen bewusst zu machen, kommen im Weiteren - unter Anwendung der Ergebnisse der dargestellten wortsemantischen Forschungsmethoden - synchronisch-vergleichende Untersuchungen zu ihrem Recht. Eine kohärente kontrastive (deutsch-ungarische) lexikalisch-semantische Analyse, vorgeführt im Begriffsbereich des 'Geschehens' (sich ereignen, geschehen, passieren, stattfinden sowie bekövetkezik, elöfordul, megesik, megtörténik, történik, végbemegy) eignet sich besonders, die semantische Breite eines Segments des Lexikons in der deutschen und ungarischen Sprache zu verdeutlichen. Der sich anschließende lexikographische 
Ausblick stellt den Lexikographen vor die mühsame Arbeit, kontrastive (deutschungarische) Wortartikel zu erstellen und damit eine noch kaum erschlossene Möglichkeit der Bedeutungsbreschreibung bzw. -darstellung auszunutzen.

Zunächst wird die Vorgeschichte des vieldiskutierten Wortfeldbegriffs abgehandelt, also von den Anfängen des Feldgedankens (Heyse, Tegnér, Abel, Osthoff, Meyer, Stöhr, Werner u.a.), über weitere Voraussetzungen seiner Entstehung (Goethe, Humboldt, Gabelentz, Saussure), bis zur ersten expliziten Formulierung des Wortfeldbegriffs durch Ipsen (1924). Untersucht der Forscher die Kontinuität der Feldforschung, kommt er zu dem Ergebnis, dass es nicht den Feldbegriff schlechthin gibt, sondern verschiedene Feldbegriffe mit jeweils verschiedenen Voraussetzungen und Zielsetzungen. In erster Linie wird deshalb eine Übersicht über die einzelnen Ausprägungsvarianten der Wortfeldtheorie in chronologischer Reihenfolge gegeben, und es werden auch die wichtigsten kritischen Einwände gegen sie vorgebracht. Nach einer kurzen Erörterung und Würdigung von drei frühen Versionen des Feldbegriffs - Ipsens 'formal-inhaltliche Felder' (1932), 'elementare Bedeutungsfelder' von Porzig (1934) und 'Minimalfelder' von Jolles (1934) - wird bei der Erörterung der Feldlehre(n) als nächster Schritt auf ihre Hauptvertreter eingegangen, auf Trier, den Vertreter des paradigmatischen lexikalischen Feldes, und auf Weisgerber, der diese Lehre im Rahmen der inhaltbezogenen Grammatik weiterentwickelt hat. Die Explikation dieser zwei Ausprägungsvarianten der Feldtheorie erfolgt in vier Arbeitsphasen. Zunächst wird davon ausgegangen, wie sie den Feldbegriff definierten, wobei auch aufdie Frage der Terminologie eingegangen wird. Im Weiteren wird dieStruktur des vorgeschlagenen Feldes oft durch Vergleiche mit anderen Feldauffassungen erörtert, um die Theorie der Feldlehre gegen andere Forschungsrichtungen (Ipsen, Porzig, Jolles, Wartburg) abzugrenzen und sie dem Leser angemessen vorzustellen. Im dritten Schritt wird die Anwendung des jeweiligen Feldbegriffs an konkreten Beispielen exemplifiziert und abschließend werden auch Kritikpunkte an dem gegebenen Feldbegriff angeführt, weil kritische Stellungnahmen zu seiner Weiterentwicklung wesentlich beitragen. Um die Kontinuität der Wortfeldforschung zu demonstrieren, werden im Folgenden neben der klassischen Feldauffassung Ipsen-Trierscher und Trier-Weisgerberscher Prägung zahlreiche neuere, linguistisch ebenfalls relevante Forschungsarbeiten der 50er und 60er Jahre behandelt - Porzig (1950), Öhmann (1953), Schwarz (1959), Kandler (1959), Gipper (1959), Schwarz (1966), Trier (1968), Ducháček (1968). Seit Beginn der 60er Jahre erfährt die Theorie des lexikalischen Feldes durch die seit dieser Zeit aufkommende semantische Inhaltsanalyse eine weitgehende Neufassung. Ins Blickfeld kommt noch ein wesentlicher Faktor, nämlich die syntaktische Komponente der Sprachbetrachtung und dadurch die Untersuchung des Verhältnisses zwischen Paradigmatik und Syntagmatik. Diese Entwicklungsphase der Wortfeldtheorie in den 60er und 70er Jahren wird durch den strukturalistischen Neuansatz und durch die Einbeziehung der Syntagmatik geprägt. Besonders beachtenswert sind auch die Entwicklungen der letzten zwei Jahrzehnte (Lutzeier, Helbig, Schumacher, Schreiber, Sommerfeldt, Starke, Geckeler, Weber, Busch, Wotjak, Busch, Hundsnurscher). Daran anschließend wird die Aufmerksamkeit auf mögliche Systematisierungen der von den einzelnen Wissenschaftlern vorgeschlagenen 
Feldern gelenkt, auf die von Weisgerber (1939), Porzig (1950), Ducháček (1968), Sčur (1977), Kertscheff (1977), Schippan (1987) und S. Piehler (1996).

Des Weiteren wird die Frage gestellt, was unter Wortfeld zu verstehen ist. Als Untersuchungsgegenstand werden hier deutsche und ungarische 'geschehen'-Verben ausgewählt. An dieser Stelle wird auch der Begriff 'Geschehen' definiert. Das zu untersuchende Wortmaterial wird vor allem durch den onomasiologischen Ansatz konstituiert. Zur Ermittlung des Forschungsmaterials braucht man einerseits das in Wörterbüchern objektivierte Wissen um den Sprachgebrauch, andererseits nutzt man auch seine Beobachtungen aus dem Sprachgebrauch. Sowohl Wörterbucharbeit als auch Selbstbeobachtungen und Informantenbefragung sind integrative Teile der lexikalisch-semantischen Untersuchung. Einer alphabetischen Auflistung der zum Begriffsgebiet gehörenden Lexeme kann man jedoch nicht viel über die inhaltlichen Beziehungen entnehmen. Das onomasiologische Vorgehen erlaubt keinen Einblick in Fügungspotenzen, Distributionen, also in den semantischen Aufbau der gegebenen Benennungseinheiten. Anhand dreier umfangreicherer deutscher semasiologischer bzw. zweier ungarischer erläuternder Wörterbücher wird an zwei deutschen (sich ereignen, passieren) bzw. ungarischen 'geschehen'-Verben (bekövetkezik, végbemegy) veranschaulicht, wie uneinheitlich die Bedeutungsbeschreibung und -darstellung in den einzelnen Wörterbüchern erfolgt. Die angeführte Vielfalt an Synonymen, Paraphrasierungen, Textbeispielen und literarischen Zitaten wie auch die unterschiedliche Anzahl der Wörterbucheintragungen und Bedeutungsschattierungen bringt die kontrastive lexikalisch-semantische Analyse eines Bereichs der zusammengestellten Feldkandidaten nicht weiter. Als Lösung bietet sich dafür an, in einer weiteren Arbeitsphase eine eigene semantische Deskriptionsmethode für verbale Glieder des Wortfeldes 'Geschehen'/ 'történés' auszuarbeiten.

Im Folgenden stellt sich die Frage, welche neuen Beschreibungsaspekte die kurrenten wortsemantischen Konzeptionen für die Wortfeldtheorie bringen. Mein Anliegen ist einerseits die zusammenfassende Darstellung und Beurteilung der gängigen wortsemantischen Theorien, andererseits die Dokumentation ihrer Ergebnisse an einem konkreten Wortfeld. Folgende Auffassungen werden - der Reihenfolge nach - einer Untersuchung unterzogen: der Wortfeldbegriff von Lutzeier, die Merkmalssemantik und die Prototypensemantik. Es stellt sich hier heraus, was in diesen Auffassungen hinsichtlich der vertiefenden lexikalisch-semantischen Analyse der Wortfeldstruktur gut anwendbar bzw. irrelevant ist. Die zentrale Fragestellung lautet nicht W o r t f e l d t h e o r i e - pro oder kontra? bzw. Merkmalssemantik, ja oder nein?, sondern Wortfeldsemantik - ja, aber wie?

Abschließend werden Stand und Aufgaben der lexikalischen Forschung behandelt. In Form eines Exkurses wird über die Abgrenzung und valenzlexikographische Beschreibung der 'geschehen'-Verben auf der Grundlage der Forschungsliteratur ein Überblick gegeben. Nach der Vorstellung der Arbeitsmethode für die kontrastive lexikalisch-semantische Analyse erfolgt die Auswahl der zu analysierenden deutschen bzw. ungarischen Verben. 
Daran anschließend werden die Wortfeldglieder im Deutschen und Ungarischen (sich ereignen, geschehen, passieren, stattfinden und bekövetkezik, elöfordul, megesik, megtörténik, történik, végbemegy) verglichen.

\section{Wissenschaftliche Ergebnisse der Forschungsarbeit}

Den Rahmen für den kontinuierlichen Entwicklungsprozess des Wortfeldbegriffs bieten die Anfänge der Bedeutungs- und Bezeichnungslehre und die Auffassung der Wortfelder als kognitive Orientierungspunkte (Lutzeier 1993, Tóth 2001). Die Darstellung der Wortfeldlehre(n) in ihrer Theorie und praktischen Anwendung fällt umfangreich aus, weil im Gegensatz zu vielen anderen Forschungen nicht nur auf die Auffassungen ihrer Hauptvertreter, sondern auch auf ihre weiteren Ausprägungen eingegangen wird. So kann ein verhältnismäßig vollständiger Einblick in die Entstehungs- und Entwicklunggeschichte des Wortfeldgedankens gewonnen werden.

Auf der Grundlage der umfangreichen Fachliteratur ist festzustellen, dass es sich um eine Theorie handelt, die immer wieder neu diskutiert wird. Der Forscher findet so verschiedene Feldbegriffe mit jeweils verschiedenen Voraussetzungen und Zielsetzungen. In meinem Periodisierungsvorschlag werden sechs Phasen der Theorieentwicklung herausgearbeitet:

1. Die Anfänge der Theorientwicklung

2. Die frühe Phase der Wortfelddiskussion

3. Die dritte Phase: umfangreiche Felduntersuchungen 1931-1938

4. Die vierte Phase der Wortfelddiskussion 1939-1968

5. Eine Sonderphase der Wortfelddiskussion: die 60er und Anfang der 70er Jahre

6. Entwicklungen der letzten zwei Jahrzehnte (vgl. Tóth 2001: 89ff.).

Es kann nachgewiesen werden, dass die sog. Stunde-Null in der Feldforschung nicht die Trierschen Ansätze bedeuten, sondern das zeitlich getrennte Aufkommen der anfangs vorwiegend historisch orientierten Bedeutungs- und Bezeichnungslehre. Die unterschiedlichen Feldauffassungen entstehen im Grunde genommen in Opposition dazu. Das Gedankengut der neuen Alternative zu der Semasiologie, die das isolierte Wort untersucht, lässt sich in zahlreichen nicht alphabetischen, systematischen Sachoder Begriffswörterbüchern verfolgen. Es ist wichtig, auch hier zu betonen, dass die Darstellung der Bedeutungsverwandtschaft in der Lexik durch die Entwicklung der Onomasiologie neue Impulse erhält.

Die erste Phase der Theorieentwicklung wird des Weiteren durch die Neuorientierung der gesamten Sprachwissenschaft bei Saussure bzw. v. d. Gabelentz und folglich durch ihren entscheidenden Einfluss auf die Entstehung der verschiedenen Richtungen der Wortfeldlehre geprägt. Bestimmend sind hier außerdem auch die Auswirkungen anderer Wissenschaftsdisziplinen auf die Gesamtentwicklung der Sprachwissenschaft des 20. Jahrhunderts, insbesondere der zwanziger und dreißiger Jahre. Hervorgehoben werden davon die ganzheitlichen Methoden in der Psychologie. Wichtig ist auch die Kritik an 
der Semasiologie und Onomasiologie v.a. von Weisgerber, weil es dadurch möglich wird, die sprachlichen Inhalte als selbständiges Forschungsobjekt zu erkennen. Die erste Phase der Theorieentwicklung wird durch die drei ältesten Ansatzpunkte zu den Feldlehren (Heyses Analyse des Wortfeldes 'Schall', Osthoffs Gedanken des Suppletivwesens und Meyers Bild eines Bedeutungssystems) sowie durch die Vorstellung des historischen Kontextes des metaphorisch verwendeten Ausdrucks Feld im Bereich der Psychologie und Physik abgerundet.

Da drei frühe Varianten des Bedeutungsfeldes (Ipsens 'formal-inhaltliche Felder', Porzigs 'Wesenhafte Bedeutungsbeziehungen' und Jolles 'Minimalfelder') m.E. im Gegensatz zur Ansicht in der Fachliteratur - in der weiteren Feldforschung eine nennenswerte Wirkung ausüben, ist eine zweite Phase abzusondern. Nur durch die kontinuierliche Beschäftigung mit dem Feldgedanken können sich immer wieder neuere Feldanschauungen mit Erfolg durchsetzen.

Die dritte Phase, in der 1931-1938 schon umfangreichere Felduntersuchungen entstehen, ist durch den Trierschen paradigmatischen lexikalischen Feldbegriff und die Abgrenzung gegen andersartige Feldbegriffe von Ipsen, Porzig und Jolles gekennzeichnet. Ihre Arbeiten sind wegweisend für die weitere Entwicklung des Feldgedankens. Hier muss die Triersche Feldlehre auch gegen die von Wartburg vorgeschlagene sprachpsychologische Lösung des Diachronie-Synchronie-Problems abgegrenzt werden, denn die Trierschen Untersuchungen richten sich nach dem sprachinhaltlichen und geistigen Unterschied zwischen dem alten und dem neuen Feldgefüge. Auch die lebhafte Kritik an Triers Feldauffassung darf nicht verschwiegen werden. Die Argumente der Kritiker beziehen sich auf die theoretischen Fragestellungen und nicht auf die dazugehörigen praktischen Ausführungen.

Infolge der Revision des eigenen Wortfeldmodells seitens Trier schon 1934, der Rehabilitation des Ipsenschen Feldbegriffs von Schwarz 1969 und der gerechteren Würdigung des Ipsenschen Beitrags zur Entwicklung der Wortfeldtheorie seitens Trier 1968 wird die Worfeldtheorie Anfang der 30er Jahre als das Ipsen-Triersche Wortfeldmodell, in den späteren 30er Jahren als das Trier-Weisgerbersche Modell apostrophiert. Der letztere Wortfeldbegriff deutet schon auf die nächste, vierte Phase der Theorieentwicklung hin, in der der Begriff des Feldes von Weisgerber mit großer Begeisterung übernommen und in der inhaltbezogenen Grammatik weiterentwickelt wird. Neben dem Ausbau einer neuen Feldmethode, in der der Triersche paradigmatische Ansatz mit einem syntaktischen verbunden wird, und der Typologisierung der Wortfelder, besteht Weisgerbers Verdienst vor allem darin, dass er die Feldlehre in ein umfassendes sprachtheoretisches Gebäude einordnet. Nicht vergessen darf man auch Reuning, der Anfang der 40er Jahre den Versuch unternimmt, auch selbst ein umfangreiches Feld, das Feld der angenehmen Gefühle in der deutschen und englischen Gegenwartssprache zu vergleichen. Um die Weiterentwicklung der Theorie und Praxis der Wortfeldlehre in ihrer Komplexität darzustellen, dürfen weder neuere Auffassungen der Protagonisten der Wortfeldtheorie (Trier, Porzig) noch Forschungsergebnisse zahlreicher weiterer Theoretiker in den 50er 
und 60er Jahren (wie z.B. Öhmann, Kandler, Gipper etc.) unerwähnt bleiben. Dank der intensiven Bemühung um die Präzisierung und Weiterentwicklung der älteren Feldkonzeption von Schwarz, einem Schüler von Trier, wird dieses Modell in Anlehnung an Herbermann (1995) als Trier-Schwarzsche Konzeption bezeichnet.

Die Wortfelddiskussion in den 60er Jahren und Anfang der 70er Jahre wird als eine Sonderphase bezeichnet, weil sie revolutionäre Neuerungen aufweist. Diese fünfte Entwicklungsphase der Wortfeldkonzeption wird durch den methodischen Neuansatz von der strukturalistischen Sprachwissenschaft und durch die Einbeziehung syntaktischer Komponenten geprägt. Durch Coserius strukturelle Wortfeldmethode erlebt der Terminus Wortfeld in den ausgehenden 60er und beginnenden 70er Jahren eine Renaissance. Coserius Wortfeldmodell basiert auf dem Prinzip der funktionellen Opposition und der Annahme der rein kompositionellen Bedeutungskonstitution. In dieser Entwicklungsphase besteht das Verdienst von Geckeler, dem Schüler Coserius, darin, dass er die wichtigsten Begriffe und Elemente für die Inhaltsanalyse in unterscheidende Züge bereitstellt. In Grebes satzstrukturbezogener Konzeption bedeuten in dieser Sonderphase die Begriffe Sinnkopplung und Worthof einen Rückgriff auf den Porzigschen Feldbegriff.

Die 70er Jahre sind charakterisiert durch ein geringes Interesse für die Problematik der Wortfeldtheorie. Lutzeier war es, der das lange Schweigen der Wortfeldtheoretiker unterbricht und 1981 eine eigenständige Wortfeldkonzeption erarbeitet. Das Lutzeiersche Modell leitet in meiner Darstellung die sechste und letzte Entwicklungsphase ein (Lutzeier 1981). Seit den 80er Jahren kommt es zu einer Wiederbelebung und Erneuerung der Wortfeldtheorie durch Helbig, Schumacher, Schreiber, Sommerfeldt, Starke u.v.a. Wortfelder werden mit unterschiedlichen linguistischen Methoden beschrieben. Um die Kontinuität der Wortfeldforschung deutlich zu machen, ist es äußerst wichtig, auch neuere Forschungsergebnisse der 90er Jahre, wie z.B. die von Piehler, Lutzeier, Geckeler, Dupuy-Engelhardt, Weber, I. Busch, Emsel, Wotjak, H-J. Busch, Hundsnurscher hervorzuheben (vgl. dazu Lutzeier 1993). Da die Wortfeldproblematik in der Linguistik der 90er Jahre kein abgeschlossenes Kapitel darstellt, ist der Autor dieses Beitrags davon überzeugt, dass in diesem Bereich weitergeforscht werden muss. So lassen weitere neuere Forschungergebnisse hoffentlich nicht lange auf sich warten.

Die Bedeutungen der lexikalischen Einheiten werden als dynamisch, veränderlich und in einem gewissen Sinne als vage angesehen. Die Bedeutungen von Lexemen, da sie in unserem mentalen Lexikon nicht isoliert, sondern vernetzt abgespeichert sind, stehen in vielfältigen Beziehungen $\mathrm{zu}$ den Bedeutungen anderer Lexeme. Aufgrund dieser semantischen Verbindung sind die Lexeme jeder Sprache globalen Organisationseinheiten, den Wortfeldern, zuzuordnen. Wortfelder als sprachliche Ausprägungen des Sinnbezirks stellen Modelle für eine effiziente Wissensaufbereitung über Realitätsbereiche dar. Unter einem Wortfeld wird ein solches lexikalisches Paradigma verstanden, das eine Vielzahl von bedeutungsverwandten Lexemen eines gemeinsamen Referenzbereichs einschließt. 'Geschehen' wird als kontinuierlicher 
Verlauf von Vorgängen, Ereignissen oder als Bruch in dieser Kontinuität definiert.

Im Laufe der weiteren Forschungsarbeit wird zum einen genügend verbales Material für die sprachlichen Ausprägungen des Begriffsbereichs 'Geschehen' im Deutschen und Ungarischen ermittelt, zum anderen werden erste Einblicke in die Bedeutungsstruktur einiger ausgewählter Benennungselemente gewährt. Im Zusammenhang mit der onomasiologischen Aufbereitung der Wortlisten ist zu beachten, dass eine definitive Aufzählung der dazugehörigen Lexeme nicht zu erwarten ist. So sieht sich der Forscher mit dem Problem konfrontiert, ob sie alle in diesen Begriffsbereich gehören und ob es noch weitere Verben gibt, die ihren Platz hier haben sollten. Da dabei die Möglichkeit der ständigen Korrektur und Ergänzung in Betracht gezogen werden muss, werden für das vorliegende Begriffsgebiet einerseits mit Hilfe umfangreicher semasiologischer Wörterbücher der deutschen bzw. der ungarischen Sprache, andererseits auf der Grundlage der Forschungsliteratur noch weitere neue Glieder aufgedeckt. Neben intensiver Wörterbucharbeit sind Informantenbefragung und Selbstbeobachtungen wesentliche Bestandteile dieser - wie eigentlich jeder - lexikalisch-semantischen Untersuchung. Als Ergebnis wird eine Zusammenstellung der zum Begriffsbereich 'Geschehen' zählenden 67 deutschen und 76 ungarischen Verben gegeben (vgl. dazu Tóth 2001: 102). Eine alphabetische Auflistung der Lexeme ist nur wenig erfolgverheißend, um inhaltliche Beziehungen zu klären. Da der onomasiologische Methodenansatz keinen Einblick in den semantischen Aufbau der gegebenen Benennungseinheiten erlaubt, wird der Versuch unternommen, anhand dreier deutscher bzw. zweier ungarischer semasiologischer Wörterbücher an den Verben sich ereignen, passieren bzw. bekövetkezik und végbemegy zu exemplifizieren, wie uneinheitlich die Beschreibung und Darstellung der lexikalischen Bedeutung hier erfolgt. Die Schwächen dieser Wörterbücher machen deutlich, dass im Folgenden noch vertiefende Untersuchungen durchgeführt werden müssen. Es ist notwendig, eine eigene intra- und interlinguale semantische Beschreibungsmethode auszuarbeiten, um so einen vollkommeneren Einblick in die lexikalisch-semantische Struktur eines Bereichs der ausgefilterten deutschen und ungarischen Feldkandidaten des 'Geschehens' zu gewinnen.

Im Folgenden stellt sich die Frage, inwiefern die kurrenten wortsemantischen Auffassungen (die Konzeption Lutzeiers, die Komponential- und Prototypensemantik) zur Vervollkommnung der Methoden der Bedeutungsbeschreibung und -darstellung von Wortfeldern, insbesondere einiger inhaltsarmer 'geschehen'-Verben, beizutragen vermögen. Als Ergebnis ist neben der zusammenfassenden Darstellung und Beobachtung dieser Theorien die Dokumentation ihrer Stärken und Schwächen an konkreten Feldkandidaten des 'Geschehens' anzusehen. Lutzeier hält die Präzisierung traditioneller Auffassungen des Wortfeldbegriffs für nötig. Dass er mit einer praktikablen informellen Definition des Begriffes Wortfeld arbeitet, zeigt sich bei der Behandlung zahlreicher Beispiele für Wortfelder, die einen Überblick über ihre möglichen Strukturen bieten. Beim Analyseversuch der'geschehen'-Verben mit Lutzeiers Methode ergeben sich allerdings u.a. im Zusammenhang mit dem verbalen Kontext, mit der Wahl der semantischen Aspekte und mit den Dimensionen Schwierigkeiten. Bei der Erörterung der Theorieentwicklung 
der semantischen Konstituentenbetrachtung mit besonderer Berücksichtigung der unterschiedlichen Möglichkeiten der Darstellung der Analyseergebnisse (wie z.B. der Matrix- oder Baumgraphendarstellung und der Klammerschreibung) zeigt sich, dass die semantische Komponentenanalyse für die Bedeutungsbeschreibung von Autosemantika, allerdings nur für bestimmte Wortschatzbereiche, besonders gut geeignet ist. Im Zusammenhang mit der Durchführung von praktischen Sem-Analysen sind einige schwerwiegende Probleme zu nennen: z.B. die Zahl der Seme, die Bezeichnung der Seme und die Ermittlung der Seme. Zur Lösung des letzten Problemkreises können beispielsweise die Techniken der Bedeutungsbeschreibung der semasiologischen Wörterbücher, die eigene sprachliche Kompetenz sowie die anderer Linguisten beitragen. Die intuitive Analyse ist immer durch Informantenbefragung und experimentelle Verfahren zu ergänzen und zu kontrollieren. Zur Ermittlung der Seme ziehe ich auch zahlreiche Textbelege heran. Es gibt aber in unserem mentalen Lexikon Wörter, deren Bedeutungen mit (binären) semantischen Merkmalen oder mit elementaren Prädikaten und Argumenten nicht exhaustiv erfasst werden können, weil sie nicht distinkt sind und keine eindeutigen Grenzen haben. In diesem Fall hilft das Prototypenkonzept weiter (Kleiber 1993, Mangasser-Wahl 1996). Es kann zwar die Merkmalssemantik nicht einfach ersetzen, aber sie doch sinnvoll ergänzen.

In der Theorieentwicklung der Prototypenidee plädierte ich für ein 3-Phasen-Modell. Aus der Informantenbefragung geht klar hervor, dass der Begriffsbereich 'Geschehen'/ 'történés' über keine prototypische Kategorienstruktur verfügt. Eine adäquate Grundlage der intra- und interlingualen lexikalisch-semantischen Analyse bildet auf diese Weise der merkmalsemantische Ansatz. Die Kennzeichnung der Kernsemantik einiger ingressiven und resultativen 'geschehen'-Verben mit Hilfe von Semen hat mich davon überzeugt, dass neben den Kernsemen auch die Valenzeigenschaften der Verben in die Analyse mit einzubeziehen sind. Eine Komponentenanalyse dieser Art betrachte ich als Fortführung und Verfeinerung der Wortfeldtheorie.

Da in dem verbalen Bereich kein entsprechender Oberbegriff existiert und das Substantiv Hergang inhaltlich vage ist, wird das Substantiv Geschehen als Oberbegriff für den ganzen Begriffsbereich vorgeschlagen. Geschehen im engeren Sinne drücken m.E. die Verben aus, die den Verlauf des Geschehens kennzeichnen. Die Verben, die den Beginn bzw. das Ende oder Ergebnis des Geschehens markieren, gehören zu den 'geschehen'-Verben im weiteren Sinne.

In der Fachliteratur werden die 'geschehen'-Verben nach unterschiedlichen Gesichtspunkten abgegrenzt. Ich befürwortete eine Einteilung der deutschen Verben in 13 Subklassen nach differenzierenden Semen (vgl. Tóth 2001: 136ff.).

Was die valenzlexikographische Beschreibung und Darstellung dieser Verben betrifft, besteht unter den Linguisten kein Konsens. Ich schließe mich der Auffassung von Nikula (1995) an, betrachte aber die Verben des Geschehens nicht einwertig, sondern, weil sie in der Kommunikation oft mit notwendigen adverbiellen Bestimmungen erscheinen, in der Regel zweistellig. 
Um die geringe semantische Differenzierung der ausgewählten deutschen bzw. ungarischen Verben des Geschehensausschnittes sichtbar zu machen, wird die Technik einer intra- und interlingualen (deutsch-ungarischen) semantischen Wortfeldanalyse erarbeitet. Die Arbeitsmethode für die intralinguale Bedeutungsbeschreibung und für die kontrastive lexikalisch-semantische Analyse beruht auf der Sem-Analyse (Kernseme) und der logisch-semantischen und syntaktischen Valenzuntersuchung (Kontextseme). Die Valenzpartner werden einerseits semantisch-denotativ und semantisch-funktionell, andererseits nach ihrer morphologischen Gestalt und Satzgliedrolle charakterisiert. Letztere sind aber bei der Konfrontation nur von sekundärer Bedeutung.

Das Ergebnis der repräsentativen Informantenbefragung hat meine Annahme, dass das Feld 'Geschehen'/'történés' prototypisch aufgebaut ist, nicht bestätigt. Das mag wohl den Grund darin haben, dass die Anzahl der Befragten relativ klein war und der Begriffsbereich zu sehr abstrakter Natur ist. Deshalb habe ich im Weiteren die These über die prototypische Kategorienstruktur nicht aufrechterhalten.

Als Ergebnis der kontrastiven lexikalisch-semantischen Untersuchung des Geschehensausschnittes (sich ereignen, geschehen, passieren, stattfinden bzw. bekövetkezik, elöfordul, megesik, megtörténik, történik, végbemegy) liegen vier zusammenfassende Tabellen vor (vgl. Tóth 2001: 154ff.). Hier sind die Gemeinsamkeiten und die Unterschiede zwischen der semantischen Struktur der untersuchten deutschen und ungarischen Verben abzulesen.

\section{SChlussbemerkungen Und leXikographischer Ausblick}

Was dieZielsetzung meines Forschungsvorhabens betrifft, so wird vor allem die deskriptive Relevanz der Kontrastiven Linguistik in der lexikalischen Semantik berücksichtigt. In jeder Arbeit zur Wortfeldforschung darf natürlich ihre Praxisrelevanz sowohl im Fremdsprachen- als auch im Muttersprachenunterricht nicht vergessen werden. Die intensive Beschäftigung mit Wortfeldern dient vorzüglich zur systematischen Erfassung der sprachlichen Nuancierungen und Differenzierungen. Als gut geeignetes Mittel der Wortschatzerweiterung können sie im DaF-Unterricht in verschiedenen Übungstypen effektiv eingesetzt werden. Sie können zum einen Abschluss, zum anderen Beginn intensiver Wortschatzarbeit sein. Die mühsame Arbeit kann man mit Wortfeldern oder Teilen bestimmter Felder beginnen und danach mit komplexen funktional-semantischen Feldern fortfahren. Im Fremdsprachenunterricht können nämlich nicht nur lexikalisch zentrierte Felder unterschiedlicher Wortarten, sondern auch grammatisch-semantische Felder zur Festigung und Erweiterung des fremdsprachlichen Wissens genutzt werden. Letztere sind besonders geeignet zum Aufdecken der semantischen Beziehungen zwischen sprachlichen Einheiten verschiedener Ebenen, also zur Zusammenfassung der Systemkenntnisse. Es ist aber immer wichtig, durch die feldhafte Behandlung des Sprachsystems auch die Kommunikationsfähigkeit der Deutschlernenden weiterzuentwickeln.

Abschließend will ich die Bedeutung der Feldlehre für die Theorie und Praxis des Erstellens 
eines Wörterbuchs betonen. Es ist leicht festzustellen, dass die von der Wortfeldtheorie postulierten Strukturierungen des Wortschatzes in der praktischen Lexikonarbeit bisher kaum berücksichtigt wurden. Das hängt damit zusammen, dass die Lemmata in den einund zweisprachigen Wörterbüchern noch immer fast ausschließlich nur alphabetisch angeordnet werden. Die Benutzer zukünftiger Wörterbücher werden die Repräsentation solcher Strukturierungen immer mehr verlangen. Jedes einzelne Wort ist nämlich ein Glied im Ganzen einer Sprache. Der Wortschatz sollte deshalb in Ganzheiten, nach Wortfeldern, insbesondere nach geordneten Wortschatzbereichen, dargestellt und beschrieben werden. Wenn die Deskription in einem gegebenen Wortfeld gutfunktioniert, dann sollte sie auch in anderen Wortfeldern funktionieren. Mein Analyseverfahren lässt sich deshalb selbstverständlich auch auf die Bedeutungsexplikation und valenzmäßige Konfrontation anderer Wortfelder übertragen. Meine Bedeutungsbeschreibungsmethode bietet in der zweisprachigen (deutsch-ungarischen) Lexikographie einen neuen Weg zur Explikation der lexikalischen Bedeutung. Diese Methode könnte in den Aufbau und die Organisation deutsch-ungarischer kontrastiver Wortartikel integriert werden. Die von dem Lexikographen zu erstellenden kontrastiven Wortartikel sollten nicht in einem Wörterbuch Eingang finden, für das das Postulat der alphabetischen Lemmataordnung gilt, sondern in einem Wörterbuch, wo die Strukturierungen in Wortfeldern angeordnet werden. Die entscheidende Rolle wird dem Lexikographen zugeteilt, dem ich es zur Aufgabe setze, aus meinem Input in einem künftig zu verwirklichenden zweisprachigen (deutsch-ungarischen), nach Wortfeldern geordneten Wörterbuch kontrastive Wortartikel zu erstellen.

\section{LITERATUR}

Burgschmidt, Ernst, Dieter Götz (1974), Kontrastive Linguistik Deutsch-Englisch: Theorie und Anwendung, München: Max Hueber.

Geckeler, Horst (1971), Strukturelle Semantik und Wortfeldtheorie, München: Wilhelm Fink.

Gnutzmann, Claus (Hg.) (1990), Kontrastive Linguistik, Frankfurt/M. etc.: Peter Lang.

Harras, Gisela (Hg.) (1995), Die Ordnung der Wörter. Kognitive und lexikalische Strukturen, Berlin, New York: Walter de Gruyter.

Herbermann, Clemens-Peter (1995), "Felder und Wörter", in: Hoinkes, Ulrich (Hg.) (1995), Panorama der Lexikalischen Semantik. Thematische Festschrift aus Anlaß des 60. Geburtstags von Horst Geckeler, Tübingen: Gunter Narr, pp. 263-291.

Hoberg, Rudolf (1970), Die Lehre vom sprachlichen Feld. Ein Beitrag zu ihrer Geschichte, Methodik und Anwendung, Düsseldorf: Schwann.

Karcher, Günther L. (1979), Kontrastive Untersuchung von Wortfeldern im Deutschen und Englischen, Frankfurt/M., Bern, Las Vegas: Peter Lang.

Kleiber, Georges (1990/dt. 1993), Prototypensemantik. Eine Einführung, Tübingen: Gunter Narr.

Lee, Anthony van der, Oskar Reichmann (Hgg.) (1973), Aufsätze und Vorträge zur Wortfeldtheorie von Jost Trier, The Hague: Mouton.

Lutzeier, Peter Rolf (1981), Wort und Feld. Wortsemantische Fragestellungen mit besonderer Berücksichtigung des Wortfeldbegriffes, Tübingen: Max Niemeyer Verlag. 
Lutzeier, Peter Rolf (Hg.) (1993), Studien zur Wortfeldtheorie. Studies in Lexical Field Theory, Tübingen: Niemeyer.

Mangasser-Wahl, Martina (1996), Eine Chronologie der Entstehung und Entwicklung der Prototypentheorie, in: Mádl, Antal, Wolfgang Schmitt (Hgg.) (1996), Jahrbuch der ungarischen Germanistik, Budapest, Bonn: SCIU GmbH, pp. 83-100.

Nickel, Gerhard (Hg.) (1972), Reader zur kontrastiven Linguistik, Frankfurt/M.: Athenäum Fischer.

Nikula, Henrik (1995), "Was kann geschehen?", in: Popp, Heidrun (Hg.) (1995), Deutsch als Fremdsprache. An den Quellen eines Faches. Festschrift für Gerhard Helbig zum 65. Geburtstag, München: Iudicium, pp. 327-337.

Schepping, Marie-Theres (1982), Kontrastive semantische Analyse von Verben des Visuellen im Französischen und Deutschen, Tübingen: Gunter Narr.

Schmidt, Lothar (Hg.) (1973), Wortfeldforschung. Zur Geschichte und Theorie des sprachlichen Feldes, Darmstadt: Wissenschaftliche Buchgesellschaft.

Schwarze, Christoph, Dieter Wunderlich (Hgg.) (1985), Handbuch der Lexikologie, Königstein/Ts.: Athenäum.

Sčur, G. S. (1974/dt. 1977), Feldtheorien in der Linguistik, Düsseldorf: Schwann.

Stechow, Arnim von, Dieter Wunderlich (Hgg.) (1991), Semantik. Ein internationales Handbuch der zeitgenössischen Forschung, Berlin, New York: de Gruyter.

Tóth, József (2001), Wortfeldforschung: Entwicklungsgeschichte und kontrastive semantische Untersuchungen, Szombathely: Maedinfo (Acta Germanistica Savariensia VI. Wissenschaftliche Beiträge des Lehrstuhls für deutsche Sprache und Literatur der Hochschule "Dániel Berzsenyi“, hg. von Lajos Szalai).

Wotjak, Gerd (1971), Untersuchungen zur Struktur der Bedeutung. Ein Beitrag zu Gegenstand und Methode der modernen Bedeutungsforschung unter besonderer Berücksichtigung der semantischen Konstituentenanalyse, Berlin: Akademie-Verlag. 